\title{
The Trend of Fermented Foods
}

\section{Shue-Shue Wang1*and Chung-Chin Sun ${ }^{2}$}

${ }^{1}$ Department of Cosmetology and Health Care, Kao Yuan University, Taiwan

2Bio-Agritech Inc., 210-8877 Odlin Crescent, Richmond, BC V6X 327, Canada

*Corresponding author : Shue-Shue Wang, Department of Cosmetology and Health

\section{Mini Review}

Volume 1 Issue 1

Received Date: October 03, 2018

Published Date: October 11, 2018

DOI: $10.23880 /$ aabsc- 16000106

Care, Kao Yuan University, No. 1821, Jhongshan Rd., Lujhu Dist., Kaohsiung city, 82151, Taiwan, ROC, Email: t50037@cc.kyu.edu.tw

\section{Abstract}

Fermented food is one of the traditional human diet cultures. In early human history, it is mainly used in food preservation and taste increases. Western medicine has long been less help for some difficult chronic diseases. Some studies have shown that the gastrointestinal tract is directly related to immunity, nerves, metabolism, and the like. The microbiota of the gastrointestinal tract is closely related to health. Turning the unbalanced into balanced microbiota can often reverse the diseases and restore health, especially for chronic or degenerative diseases that are currently difficult to have drug effects, such as Parkinson's disease (PD), Alzheimer's disease (AD), depression, and cancer, etc. The discovery and research of probiotics have demonstrated their health effects by helping balance or formation of beneficial microbiota in gastrointestinal tract. Supply of probiotics has become a good way of health care in people's daily life. Fermented foods not only provide important nutrients, functional active ingredients, but also provide a group of active microorganisms that are beneficial to the body health, which can repair, balance or maintain the healthy microbiota of the gastrointestinal tract. Driven by the health care trend, fermented food has become an option for people to maintain health. Scientists have begun to interest in the feasibility of using single or combined natural herbal ferments for use in difficult diseases. Initial results have also shown significant effectiveness and they are expected to be an improvement or resolution of unresolved ill diseases in the future. In spite of fermented food or herbal medicine, it is necessary to construct a good quality of fermented food or herbal ferment. In the fermentation process, selected specific microbe or microbiota, and developed chemical and biological analysis methods to confirm the quality of the food after fermentation, and clarified the microbial population in the food after fermentation are necessary. The final products are confirmed the beneficial microbe or microbiota and show the evidence of clinical safety and efficacy. In order to widely promote the fermented foods in market in the future, the products should be cheaper than that of the current market products. One approach is researched to shorten the fermentative process which can increase inventory turnover.

Keywords: Fermented Food; Microbiota; Probiotics; Chronic or Degenerative Diseases 


\section{Introduction}

Fermented food is one of the human diet cultures. After thousands of years of human use, it has become a daily diet culture in human society. Fermented foods are initially for food preservation, and then enhance the food flavor and organoleptic properties. In the past centuries, the rapid society progress, economy growth, rising life pressure, diet imbalance, and aging population increase have led to the emergence of many chronicor degenerative diseases. In recent years, fermented foods have begun to receive more attention. The most promising food components as vitamins, minerals, fatty acids, carotenoids, polyphenols, bioactive peptides, probiotics, creatine and saponins have therapeutic potential, especially for anti-inflammatory, anti-oxidative properties and neuronal protection [1]. Plant products such as Legumes, fruit and cereals are natural sources of prebiotics. Using these plant foods as sources of fermented foods for human health products are generally used among the Asian people. It was first developed in Japan in the early days, and later the trend was sprayed to Taiwan, South Korea and China. Traditional fermented foods are solid and non-solid. Solid-state fermented foods include natto, bean curd, sauerkraut, yogurt, kimchi, pickled cucumber, fermented sausage, etc. Non-solid fermented foods are wine, beer, and other beverages. Nowadays, the awareness of health effect for fermented foods is getting better and better recognized in the society, resulting in people are willing to purchased them. The fermented food gradually becomes a life style of health care.

\section{Gut Microbiota and Health}

There is a growing interest in research to investigate the importance of gut microbiome in health and diseases. Recent studies have shown that gastrointestinal disorders are important factors. The brain-gut axis interactions are significantly modulated by the gut microbiota via immunological, neuroendocrine, and homeostasis mechanisms. Dysregulation of the brain-gutmicrobiota axis in aging [2-4], PD [5-7], AD [8-10], energy homeostasis (pathogenesis of obesity and type 2 diabetes mellitus (T2DM) $[8,10]$ is usually associated with gastrointestinal disturbance frequently preceding clinical symptoms, as well as with the pathogenesis itself. Amelioration of clinical symptoms as physical frailty, clostridium difficile colitis, vulvovaginal atrophy, colorectal carcinoma, and atherosclerotic disease of older adults by manipulating microbiota and microbiome of older adults can be innovative strategy [3]. There is increasing interests that are the gut microbiome in health and diseases [8] and interaction between host genetics and gut microbiota [2]. The change in gut microbiota and colonic gene expression is obviously related to aging, especially the strong correlations between specific bacterial genera and host gene expression [4]. Imbalances in the gut microbiotacan induce inflammation that is associated with the pathogenesis of obesity, type 2 diabetes mellitus, and $\mathrm{AD}[10]$.

\section{Probiotics}

In the recent years, the health care of the gastrointestinal tract has become the focus of attention. Microbiota balance or probiotics enhancement can be beneficiary to health. Some studies have shown that probiotics have a significant improvement in chronic or degenerative diseases [11-16]. The prebiotics and probiotics can maintain a healthy microbiome or restore balance [17]. At present, Biotech companies provides the market with a variety of different probiotics, either one single or various prescribed probiotics combination in beverage or various dosage forms as powders or granules. One of the main formulas of probiotics, provided by Biotech companies', is synbiotics, which use selected probiotics with their prebiotics, for use in humans or animals. Prebiotics are usually chosen foods for probiotics in order to help proliferating probiotics in the digestive tracts. The general public also began to try to solve some life problems, such as constipation, allergies and so on, with probiotics.

\section{Fermented Foods and Health}

Except increase of food shelf life, safety, and organoleptic properties, the fermented foods have enhanced nutritional and functional properties and produced a versatile source of bioactive molecules and bioactive microbes during fermentation process. The fermented food with bioactive microorganisms may improve gastrointestinal health and provide health benefits, including lowering the risk of type two diabetes and cardiovascular diseases [18-21].

\section{Safety in Microbiota of Fermented Foods}

Many traditional fermented foods used the nature fermentation are in market. The difference in the microbiota of traditional fermented foods varies with the geographical origin and manufacturing process. If the food is spontaneously fermented that the bacteria are from food itself or environment, the microbiota of fermented foods is unclear and often presents diverse developments [22-24]. Fermented foods are generally produced using a starter, a dominant microbiota from reused fermentation material, that has been demonstrated to become clear and 


\section{Annals of Advanced Biomedical Sciences}

stable microbiota. The traditionally fermented foods with selected microbes lay a foundation for manipulating the process of fermentation and for improving the overall quality of fermented products while retaining their traditional traits [23]. In order to have tractable model ecosystems, the microbial communities of fermented foods can be manipulated with selected or serial transfer microbes with microbial growth variables such as temperature, salinity, and moisture results to generate distinct microbial communities [25]. The safety of fermented food should be further discussed and the product specification and quality regulation should be established.

\section{Cheaper Production}

The production process of fermented foods for fruits and vegetables often requires longer time, which makes the production cost of good fermented foods high, resulting in excessive prices to obstruct its universalization. It is important to research shortening the fermentation process to reduce the cost in order to widely promote the fermented foods in public

\section{Medical Herbal Plant Fermentation}

Traditional Chinese medicine can improve the structure of gut microbiota by increasing probiotics and reducing pathogens for the prevention and treatment of diseases, including obesity and inflammation, insulin resistance, type 2 diabetes, non-alcoholic fatty liver disease, inflammatory bowel disease and other diseases [26]. Fructooligosaccharides (prebiotics) extracted from Morinda officinalis (OMO) are found to exert effective memory improvements in AD-like animals, by maintaining the diversity and stability of the microbial community [9]. It implies that the therapeutic effect of the traditional medicine, OMO, can improve the various neurological diseases such as AD.In China's folk usage, there have been long-term use of fermented Chinese herbal medicine to treat some ill diseases. At present, there are large-scale fermented products of Chinese herbal medicines or plants to be used in chronic diseases treatment in China, and have shown significant results. It is expected that there are more herbal plant ferments that will be involved in researches or clinical trials to solve unmet diseases.

\section{Conclusion}

Fermented foods can be functional foods for health care or disease prevention. Pharmaceutical-grade fermented food can be for ill diseases treatment. It is still waiting to solve the problems as confirm the safe and beneficial microbiota as probiotics, shorten the fermentation process to reduce the production cost, raise scientific evidence for safety and effective use. In future, the herbal fermented products with regulatory classification may serve as a treatment or relief for many of the chronic diseases that are currently unsolved solution.

\section{Acknowledgement}

I am grateful to my current and past colleagues for their study, research and promoting fermented food in Taiwan

\section{References}

1. Frutos MJ, Valero-Cases E, Rincon-Frutos L (2018) Food components with potential to be used in the therapeutic approach of mental diseases. Curr Pharm Biotechnol.

2. Patrignani P, Tacconelli S, Bruno A (2014) Gut microbiota, host gene expression, and aging. J Clin Gastroenterology 48(1): S28-31.

3. Zapata HJ, Quagliarello VJ (2015) The microbiota and microbiome in aging: potential implications in health and age-related diseases. J Am Geriatr Soc 63(4): 776781.

4. Van der Lugt B, Rusli F, Lute C, Lamprakis A, Salazar E, et al. (2018) Integrative analysis of gut microbiota composition, host colonic gene expression and intraluminal metabolites in aging C57BL/6J mice. Aging (Albany NY) 10(5): 930-950.

5. Mulak A, Bonaz B (2015) Brain-gut-microbiota axis in Parkinson's disease. World J Gastroenterol 21(37): 10609-10620.

6. Scheperjans F, Aho V, Pereira PA, Koskinen K, Paulin L, et al. (2015) Gut microbiota are related to Parkinson's disease and clinical phenotype. Mov Disord 30(3): 350-358.

7. Heintz-Buschart A, Pandey U, Wicke T, Sixel-Döring F, Janzen A, et al. (2018) The nasal and gut microbiome in Parkinson's disease and idiopathic rapid eye movement sleep behavior disorder. Mov Disord 33(1): 88-98.

8. Naseer MI, Bibi F, Alqahtani MH, Chaudhary AG, Azhar EI, et al. (2014) Role of gut microbiota in obesity, type 2 diabetes and Alzheimer's disease. CNS Neurol Disord Drug Targets 13(2): 305-311. 
9. Chen D, Yang X, Yang J, Lai G, Yong T, et al. (2017) Prebiotic Effect of Fructooligosaccharides from Morinda officinalis on Alzheimer's Disease in Rodent Models by Targeting the Microbiota-Gut-Brain Axis.Front Aging Neurosci 9: 403.

10. Jiang C, Li G, Huang P, Liu Z, Zhao B (2017) The Gut Microbiota and Alzheimer's Disease J Alzheimers Dis 58(1): 1-15.

11. Go J, Kim JE, Kwak MH, Koh EK, Song SH, et al. (2016) Neuroprotective effects of fermented soybean products (Cheonggukjang) manufactured by mixed culture of Bacillus subtilis MC31 and Lactobacillus sakei 383 on trimethyltin-induced cognitive defects mice. Nutr Neurosci 19(6): 247-59.

12. Takada M, Nishida K, Kataoka-Kato A, Gondo Y, Ishikawa H, et al. (2016) Probiotic Lactobacillus casei strain Shirota relieves stress-associated symptoms by modulating the gut-brain interaction in human and animal models. Neurogastroenterol Motil 28(7): 1027-1036.

13. Kuo PH, Chung YE (2018) Moody microbiome: Challenges and chances. J Formos Med Assoc S09296646(18): 30623-30625.

14. Nazir Y, Hussain SA, Abdul Hamid A, Song Y (2018) Probiotics and Their Potential Preventive and Therapeutic Role for Cancer, High Serum Cholesterol, and Allergic and HIV Diseases. Biomed Res Int 2018: 3428437.

15. Scott AJ, Merrifield CA, Younes JA, Pekelharing EP (2018) Pre-, pro- and symbiotics in cancer prevention and treatment-a review of basic and clinical research. Ecancermedicalscience 12: 869.

16. Vareki SM, Chanyi RM, Abdur-Rashid K, Brennan L, Burton JP (2018) Moving on from Metchnikoff: thinking about microbiome therapeutics in cancer. Ecancermedicalscience 12: 867.

17. Quigley EMM (2018) Prebiotics and Probiotics in Digestive Health. Clin Gastroenterol Hepatol S15423565(18): 31019-X.
18. Marco ML, Heeney D, Binda S, Cifelli CJ, Cotter PD, et al. (2017) Health benefits of fermented foods: microbiota and beyond. Curr Opin Biotechnol 44: 94102.

19. Rezac S, Kok CR, Heermann M, Hutkins R (2018) Fermented Foods as a Dietary Source of Live Organisms Front Microbiol 9: 1785.

20. Şanlier N, Gökcen BB, Sezgin AC (2017) Health benefits of fermented foods. Crit Rev Food Sci Nutr 25: $1-22$.

21. Sivamaruthi BS, Kesika P, Chaiyasut C (2018) Thai Fermented Foods as a Versatile Source of Bioactive Microorganisms-A Comprehensive Review. Sci Pharm 86(3).

22. Park EJ, Chun J, Cha CJ, Park WS, Jeon CO, Bae JW (2012) Bacterial community analysis during fermentation of ten representative kinds of kimchi with barcoded pyrosequencing. Food Microbiol 30(1): 197-204.

23. He GQ, Liu TJ, Sadiq FA, Gu JS, Zhang GH (2017) Insights into the microbial diversity and community dynamics of Chinese traditional fermented foods from using high-throughput sequencing approaches. J Zhejiang Univ Sci B 18(4): 289-302.

24. Jung MJ, Kim MS, Yun JH, Lee JY, Kim PS, et al. (2018) Viral community predicts the geographical origin of fermented vegetable foods more precisely than bacterial community. Food Microbiol 76: 319-327.

25. Wolfe BE, Dutton RJ (2015) Fermented foods as experimentally tractable microbial ecosystems. Cell 161(1): 49-55.

26. Sang TT, Guo CJ, Guo DD, Wang XY (2018) Effect of traditional Chinese medicine in inhibiting obesity and inflammatory diseases by regulating gut microbiota. Zhongguo Zhong Yao Za Zhi 43(16): 3235-3242.

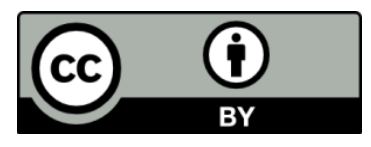

